\title{
Absence of characteristic features in two patients with inclusion body myositis
}

\author{
M F G van der Meulen, J E Hoogendijk, G H Jansen, H Veldman, J H J Wokke
}

Department of

Neurology, Division of

Neuromuscular

Disorders

M F G van der Meulen

J E Hoogendijk

$\mathrm{H}$ Veldman

J H J Wokke

Department of

Neuropathology,

University Hospital

Utrecht

G H Jansen

Correspondence to: Dr MFG van der Meulen,

Department of Neurology,

University Hospital Utrecht,

Heidelberglaan 100, 3584

CX Utrecht, The

Netherlands. Telephone

0031302507978 or 003130

2506480; fax 003130

2542100; email:

mmeulen@neuro.azu.nl

Received 11 July 1997 and in revised form 4 September 1997

Accepted 17 September 1997

\begin{abstract}
According to recently published criteria a diagnosis of definite sporadic inclusion body myositis is made if the typical histopathological abnormalities (rimmed vacuoles and abnormal accumulations of proteins, in addition to mononuclear cell infiltrates) are present. The two women described here presented with myositis which was unresponsive to treatment. Patient 1 had features of non-progressive sporadic inclusion body myositis clinically, whereas patient 2 had a very slowly progressive limb girdle syndrome. The cryostat sections of the first biopsies did not show rimmed vacuoles, even in retrospect. Only a repeated biopsy, 12 years after presentation in one patient and 18 years after presentation in the other, disclosed the typical features of sporadic inclusion body myositis. The initial absence of abnormal fibres probably represents a real absence or scarcity rather then a sampling error due to a multifocal nature of the histological abnormalities. It is of importance for the clinician to realise that some patients with myositis unresponsive to treatment, even if both clinical and histological features do not suggest sporadic inclusion body myositis, may prove to have the disease on repeated histopathological examination.
\end{abstract}

(F Neurol Neurosurg Psychiatry 1998;64:396-398)

Keywords: inclusion body myositis; diagnosis

Sporadic inclusion body myositis is a chronic inflammatory muscle disease of unknown origin. $^{12}$ Because sporadic inclusion body myositis is not responsive to corticosteroid treatment it is important to differentiate it from polymyositis at an early stage. Clinically, sporadic inclusion body myositis differs from polymyositis by the following features: duration of illness more then six months, age of onset older then 30 years, finger flexor weakness, wrist flexor more then wrist extensor weakness, prominent quadriceps weakness, and serum creatine kinase $>12$ times normal. ${ }^{3}$ Whereas both diseases have mononuclear endomysial cell infiltrates in common, sporadic inclusion body myositis is histologically differentiated from polymyositis by the presence of rimmed vacuoles and abnormal intracellular accumulations of proteins. In recently published criteria for sporadic inclusion body myositis the histopathological abnormalities are accentuated: a diagnosis of definite inclusion body myositis is made if a muscle biopsy shows mononuclear cell infiltrates, vacuoles, and either amyloid deposits or 15-18 nm tubulofilaments by electron microscopy. A diagnosis of possible sporadic inclusion body myositis is made if the clinical features are indicative but the muscle biopsy is not diagnostic. ${ }^{3}$

The patients described here show that the discriminating histopathological features, and also the typical clinical features may be absent in patients with sporadic inclusion body myositis.

\section{Case reports}

Patient 1, a 54 year old woman, presented with weakness of the facial muscles, the neck flexor muscles (MRC 4-5), the finger flexor muscles (left>right) and the iliopsoas muscles (MRC 4), which had slowly developed during the previous four years. There was no significant quadriceps weakness. The creatine kinase concentration was $413 \mathrm{U} / 1$ (normal < $100 \mathrm{U} / 1$ ). A biopsy taken from the flexor digitorum muscle showed mononuclear cell invasion of nonnecrotic muscle fibres (figure A). A diagnosis of polymyositis was made and treatment with high dose prednisone was started. A year later azathioprine was added (100 $\mathrm{mg} /$ day). In the next 10 years there was neither improvement nor progression. At the age of 66 (12 years after presentation) a second biopsy was taken. Endomysial lymphocytic infiltrates were noted, but also rimmed vacuoles in 11 of the 2500 examined muscle fibres $(0.4 \%$, figure $\mathrm{B})$. With the modified Gomori trichrome stain there was a single ragged-red fibre, but cytochrome oxidase activity was normal. Congo red staining showed intracellular amyloid deposits. The diagnosis was revised to inclusion body myositis. The cryostat sections of the first biopsy were scrutinised in retrospect, but no vacuoles were found in the 5000 muscle fibres (figure A). There were a few ragged-red fibres and cytochrome oxidase negative fibres were present. 

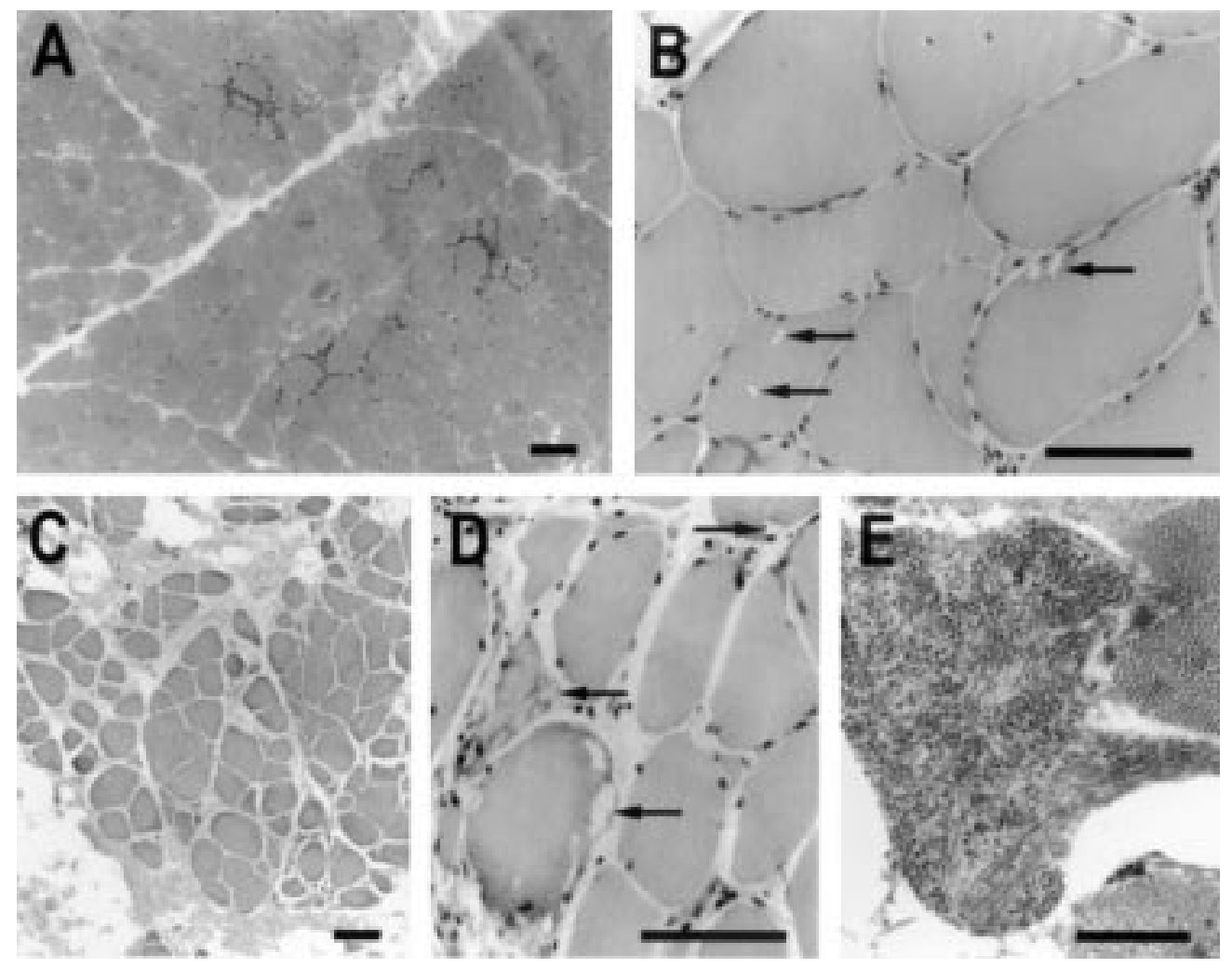

(A) Patient 1; haematoxylin and eosin stained $6 \mu \mathrm{m}$ cryostat section of the initial biopsy (flexor digitorum muscle) showing absence of rimmed vacuoles and endomysial lymphocytic infiltrates (bar $=100 \mu m$ ). (B) Patient 1; haematoxylin and eosin stained $6 \mu \mathrm{m}$ cryostat section of the second biopsy (femoral biceps muscle) showing rimmed vacuoles (arrows) (bar=100 $\mu \mathrm{m})$. (C) Patient 2; haematoxylin and eosin stained $6 \mu \mathrm{m}$ cryostat section of the initial biopsy (pectoral muscle) showing mild inflammatory changes and absence of rimmed vacuoles (bar=100 $\mu \mathrm{m}$ ). (D) Patient 2; haematoxylin and eosin stained $6 \mu \mathrm{m}$ cryostat section of the third biopsy (a brachial biceps muscle) showing rimmed vacuoles (arrows) (bar $=100$ $\mu \mathrm{m})$. (E) Patient 2; electron microscopy of the third biopsy specimen showing 16-18 nm tubulofilaments (bar $=500 \mathrm{~nm}$ ).

Patient 2, a 43 year old woman known to have scleroderma, presented with weakness of the flexor muscles of the neck and of the proximal muscles of her arms and legs (MRC 3-4), with relative sparing of the quadriceps (MRC 4-5). The symptoms had developed gradually in the previous year. The creatine kinase concentration was moderately raised at $538 \mathrm{U} / 1$ (normal<100 U/1). At that time a diagnosis of polymyositis was made and treatment with corticosteroids and azathioprine was started. However, muscle weakness gradually increased and a muscle biopsy taken at the age of 46 showed mild inflammatory changes, in line with a diagnosis of treated polymyositis (figure C). Seven years later there was facial muscle weakness in addition to the proximal weakness, but no distal weakness. A second muscle biopsy (deltoid muscle) again showed endomysial infiltrates of lymphocytes. Cyclosporin was given additionally, but the patient gradually deteriorated during the subsequent years. At the age of 61 (18 years after presentation) a third biopsy was performed. Rimmed vacuoles were seen in 15 of 1200 fibres (1\%) (figure D). No ragged-red fibres were seen with the modified Gomori trichome stain, and cytochrome oxidase activity was normal. Abundant 16-18 $\mathrm{nm}$ tubulofilaments were seen by electron microscopy (figure E). A diagnosis of sporadic inclusion body myositis was made. Shortly thereafter the patient died of an unrelated cause. On revision of the cryostat sections of the initial biopsy no vacuoles were found in 7800 muscle fibres (figure C). Revision of the cryostat sections of the second biopsy showed vacuoles in two of 1800 fibres. Neither biopsy showed ragged-red fibres or cytochrome oxidase negative fibres. Electron microscopy performed on the second biopsy was negative.

\section{Discussion}

Atypical clinical presentation as in our patient 2 is not unusual in sporadic inclusion body myositis, as recently described by Amato et al, ${ }^{4}$ and the histopathological changes are considered to be the diagnostic hallmark. ${ }^{3}$ In both of our patients, however, the initial muscle biopsies did not, even in retrospect, show the characteristic histopathological abnormalities. In some other publications reporting on the absence of rimmed vacuoles in patients with sporadic inclusion body myositis, paraffin and not cryostat material was examined. ${ }^{56}$ This is important because the typical vacuoles may be difficult to recognise in paraffin sections. ${ }^{12}$ Absence of the diagnostic abnormalities in cryostat sections has earlier been described in two others patients with definite sporadic inclusion body myositis. ${ }^{4}$ These authors suggest that a non-diagnostic biopsy is explained most likely as a sampling error due to the multifocal nature of the histopathology. However, they do not provide data on the extent of the histopathological abnormalities in the second biopsies. In the two patients described 
here, the number of abnormal fibres in the repeated biopsies was low, and therefore, we think that their initial absence reflected a real scarcity of evenly distributed vacuoles. It is noted that the disease was stable in patient 1 , only very slowly progressive in patient 2 , and that both patients were female. The relations between sex, rate of progression, and extent of histopathological abnormalities in this disease, however, remain as yet unknown.

In conclusion, in some patients with sporadic inclusion body myositis, rimmed vacuoles and other characteristic histopathological features may be scarce or absent. In patients with nonspecific clinical features sometimes only the course of the disease (unresponsiveness to corticosteroids) and a repeated muscle biopsy lead to a correct diagnosis.

1 Dalakas MC. Polymyositis, dermatomyositis and inclusion body myositis. N Engl F Med 1991;325:1487-97.

2 Mikol J, Engel AG. Inclusion body myositis. In: Engel AG, Franzini-Armstrong C, eds. Myology. New York: McGrawHill, 1994:1384-98

3 Griggs RC, Askanas V, Dimauro S, et al. Inclusion body myositis and myopathies. Ann Neurol 1995;38:705-13.

4 Amato AA, Gronseth GS, Jackson CE, et al. Inclusion body myositis: clinical and pathological boundaries. Ann Neurol 1996;40:581-6.

5 Parvis J, Fam AG, Lewis A. Clinically unsuspected inclusion body myositis. F Rheumatol 1991;18:289-92.

6 Calabrese LH, Mitsumoto H, Chou SM. Inclusion body myositis presenting as treatment-resistant polymyositis. Arthritis Rheum 1987;30:397-403. 